\title{
Electric field dependence of radiative recombination lifetimes in polar InGaN/GaN quantum heterostructures
}

\author{
Emre Sari $^{1,3,4}$, Sedat Nizamoglu ${ }^{1,3,4}$, In-Hwan Lee ${ }^{5}$ Jong-Hyeob Baek ${ }^{6}$, and Hilmi Volkan Demir ${ }^{1,2,3,4, *}$ \\ ${ }^{1}$ Department of Electrical and Electronics Engineering, ${ }^{2}$ Department of Physics, ${ }^{3}$ Nanotechnology Research Center, ${ }^{4}$ Institute of Materials \\ Science and Nanotechnology, Bilkent University, Bilkent, Ankara 06800 Turkey \\ ${ }^{5}$ School of Advanced Materials Engineering, Research Center of Industrial Technology, Chonbuk National University, Chonju 561-756, Korea \\ ${ }^{6}$ Korea Photonics Technology Institute, Gwangju 500-460, Korea \\ *Tel:[+90]( 312) 290 1021, e-mail:volkan@bilkent.edu.tr
}

We report on external electric field dependence of recombination lifetimes in polar InGaN/GaN quantum heterostructures. In our study, we apply external electric fields one order of magnitude less than and in opposite direction to the polarization-induced electrostatic fields inside the well layers. Under the increasing external electric field, we observe a decrease in carrier lifetimes $(\tau)$ and radiative recombination lifetimes $\left(\tau_{\mathrm{r}}\right)$, latter showing a weaker dependence. Our results on $\tau_{\mathrm{r}}$ show an agreement with our transfer matrix method based simulation results and demonstrate Fermi's golden rule in polar InGaN/GaN quantum heterostructures dependent on electric field.

For our study, we grew 5 pairs of $2.5 \mathrm{~nm}$ thick $\operatorname{In}_{0.15} \mathrm{Ga}_{0.85} \mathrm{~N}$ quantum well and $7.5 \mathrm{~nm}$ thick GaN barrier layers in a p-i-n diode architecture using metal-organic chemical vapor deposition (MOCVD) on a c-plane sapphire substrate. Devices with $300 \mu \mathrm{m} \times 300 \mu \mathrm{m}$ mesa size were fabricated using standard photolithography, reactive ion etching and metallization steps. We used indium-tin oxide (ITO) based semi-transparent contacts in top (p-GaN) layer for uniform application of electric field across the well layers. The fabricated devices were diced and mounted on a TOcan for compact testing.

In order to retrieve radiative recombination lifetimes, we performed steady-state and time resolved photoluminescence (PL) measurements. In time-resolved photoluminescence (TRPL) measurements, we used a pulsed InGaN/GaN laser diode with an excitation wavelength of $375 \mathrm{~nm}$. TRPL decays and intensity averaged lifetimes $(\tau)$ at $\lambda=448 \mathrm{~nm}$ (forward bias electroluminescence peak wavelength) parameterized with external electric fields are given in Figure 1a and Figure 1b, respectively. As in Figure 1b we observe a strong dependence of carrier lifetime on external electric field [1].

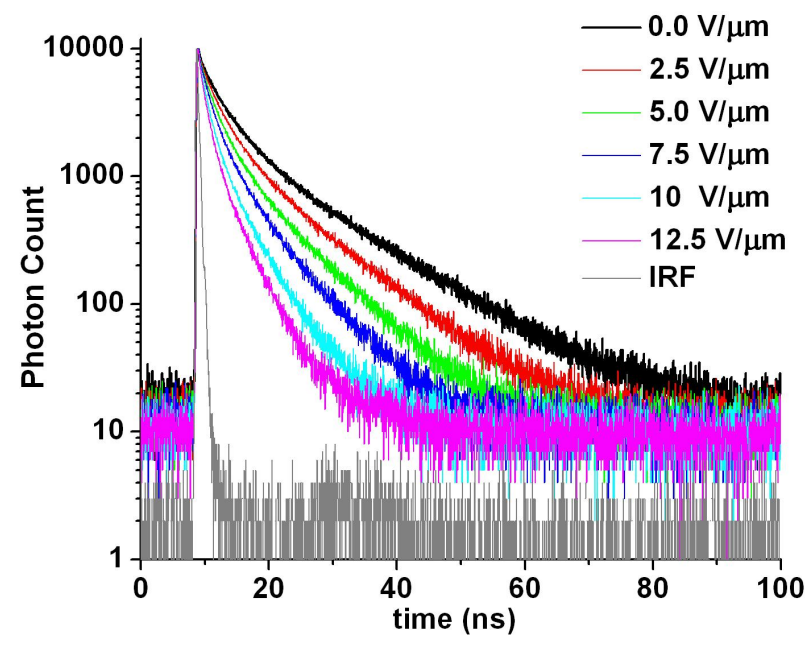

Figure 1a Time resolved photoluminescence decays of our polar InGaN/GaN quantum heterostructures.

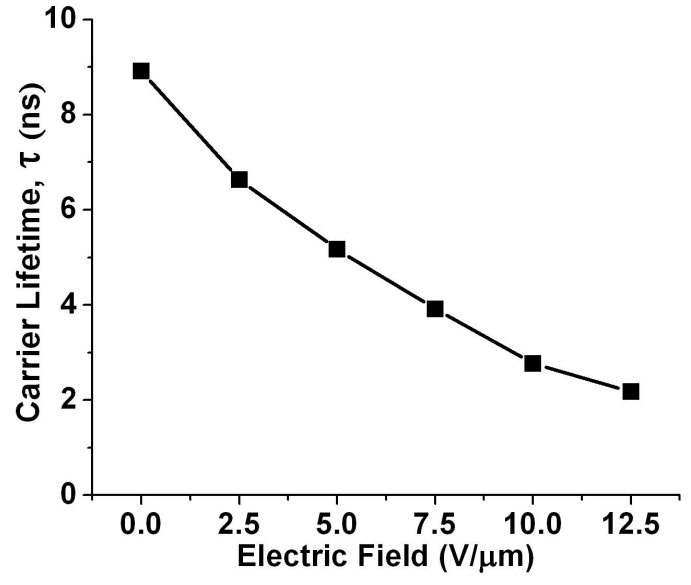

Figure 1b Carrier lifetimes dependent on the external electric fields.

We performed steady state PL measurements to relate $\tau$ and $\tau_{\mathrm{r}}$ via quantum efficiency (QE) as their ratio. In PL measurements, we used a He-Cd laser with an excitation wavelength of $325 \mathrm{~nm}$. We obtained relative quantum efficiencies by partially integrating PL spectra (between 440 and $470 \mathrm{~nm}$ ) corresponding to each external electric field level. PL spectra and relative quantum efficiencies corresponding to the same external electric field levels are shown in Figure 2b. As a result of our PL measurements, we observe a decrease in PL signal, and thus QE, together with a narrowing in the PL spectra. The narrowing is clearly seen as a blueshift in the longer wavelength part of the normalized PL spectra as in Figure $2 \mathrm{~b}$. We attribute this blueshift to reversed quantum-confined Stark effect, the increase of electron-hole transition energies with increasing external electric field [2]. 


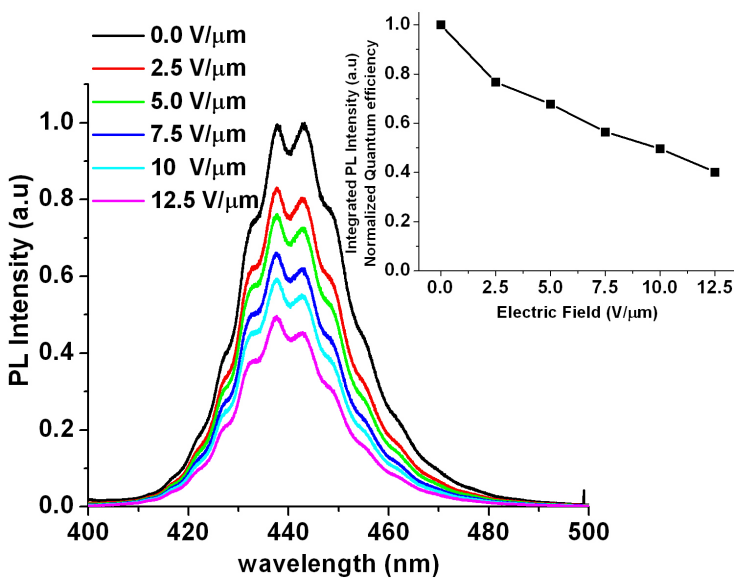

Figure 2a Steady-state photoluminescence spectra and retrieved relative quantum efficiencies of our polar InGaN/GaN quantum heterostructures.

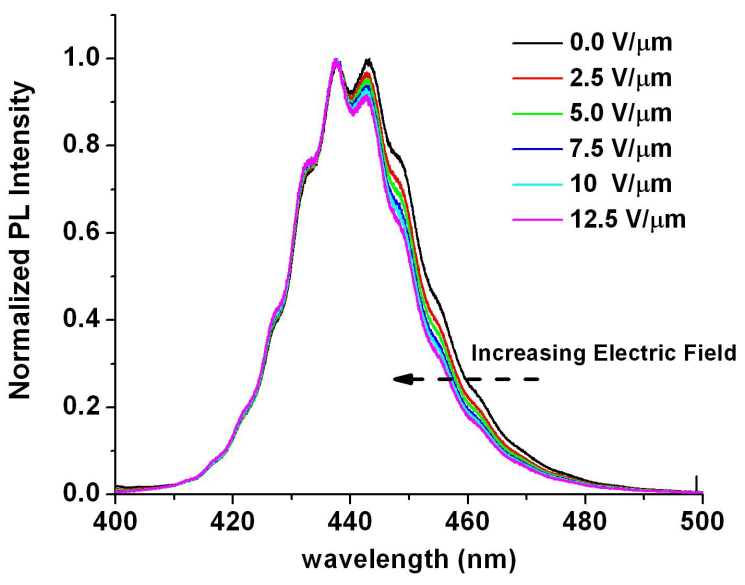

Figure 2b Normalized photoluminescence spectra our quantum structures. Longer wavelength part of the spectra exhibit a blueshift.

We obtained electric field dependence of radiative recombination lifetime from relative quantum efficiency and carrier lifetime measurements as shown in Figure 3 In the same figure, we give normalized reciprocal of squared overlap integral of calculated electron and hole ground state wavefunctions. This directly relates to radiative recombination lifetime according to Fermi's golden rule and shows the same trend as our experimental results [1].

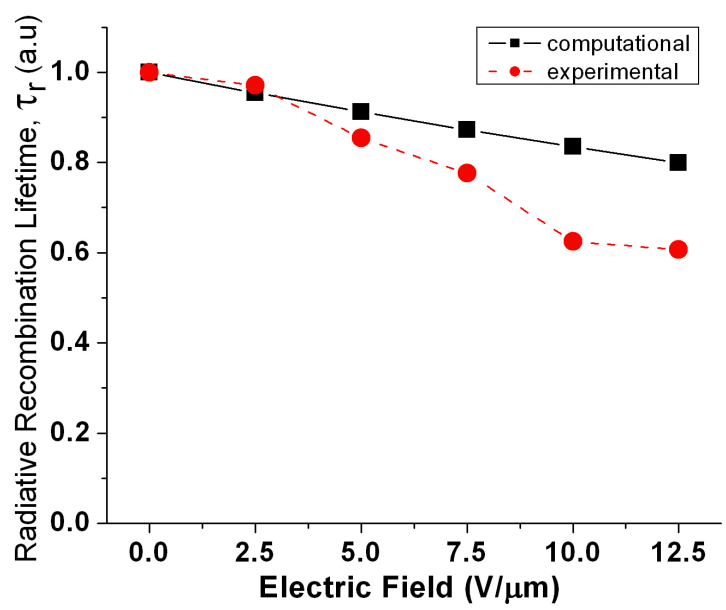

Figure 3 Relative radiative recombination lifetimes: experimental and computational results.

In conclusion, we studied electric field dependence of radiative recombination lifetime $\left(\tau_{\mathrm{r}}\right)$ in polar InGaN/GaN quantum heterostructures. We experimentally and theoretically showed that $\left(\tau_{\mathrm{r}}\right)$ decreases with increasing external electric field. As a result of our study, we demonstrated Fermi's golden rule in polar InGaN/GaN quantum heterostructures [1].

Acknowledgements: This work is supported by EU MOON, EU NOE PHOREMOST and TUBITAK 104E114, 106E020, 105E065, and 105E066. H.V.D. and E.S. also acknowledge additional support from Turkish Academy of Sciences (TUBA GEBIP) and TUBITAK.

References

[1] E. Sari, et al., Appl. Phys. Lett., 94, 211107 (2009).

[1] E. Sari, et al., Appl. Phys. Lett., 90, 011101 (2007). 